Sharif University of Technology
Scientia Iranica
SCIENTIA

\title{
Analytical solutions to nonlinear oscillations of micro/nano beams using higher-order beam theory
}

\author{
M.M. Roozbahani, N. Heydarzadeh Arani, M. Moghimi Zand* and \\ M. Mousavi Mashhadi \\ School of Mechanical Engineering, College of Engineering, University of Tehran, Tehran, Iran.
}

Received 1 May 2015; received in revised form 27 September 2015; accepted 26 December 2015

KEYWORDS
MEMS;
NEMS;
Higher-order beam
theory;
High thickness;
Analytical solutions;
Nonlinear vibrations.

\section{Introduction}

One of the most significant phenomena associated with micro/nano electromechanical systems, actuated by electrostatic forces, is known as pull-in instability phenomenon, which has been investigated by many researchers. Nathanson et al. [1] and Taylor [2] reported pull-in instability in the $1960 \mathrm{~s}$. Microbeams actuated with electrostatic forces are widely used for different applications such as signal filtering and mass sensing [3].

When the applied voltage exceeds a critical value, called pull-in voltage $\left(V_{p i}\right)$, the flexible microbeam

\footnotetext{
*. Corresponding author. Tel.: +98216111480\%: Fax: +982188013029 E-mail address: mahdimoghimi@ut.ac.ir (M. Moghimi Zand)
}

deflects toward the rigid plate. In microbeams, pull-in instability continues to become increasingly important for the design of electrostatic MEMS and NEMS devices. Pull-in analysis can be categorized into two groups according to its state. When the rate of voltage variation is low, inertia has no effect on the microsystem behavior and the critical value of voltage is known as static pull-in voltage $\left(V_{p i}\right)$. In contrast, when the rate of voltage variation is considerable, the effect of inertia must be taken into account and the critical voltage value is called dynamic pull-in voltage $\left(V_{\text {pid }}\right)$. The pull-in instability associated with this situation is called dynamic pull-in instability [4,5]. More details about this phenomenon are given in [6-14]. In most nonlinear studies, structures are modeled by Euler-Bernoulli theory. Moghimi Zand et al. obtained pull-in voltage of a microbeam considering the EulerBernoulli beam theory [15]. In another study, Moghimi 
Zand et al. studied nonlinear frequency of the nonlinear vibration of a microbeam considering Euler-Bernoulli beam theory [16]. The large deformation of an EulerBernoulli beam subjected to an arbitrary distributed load was investigated by Malekie et al. [17]. Sedighi et al. investigated the nonlinear dynamics of a Nanobridge pull-in instability considering the centrifugal force and the rarefied gas flow [18]. Farrokhabadi et al. analyzed the influence of the Casimir force on pull-in instability of nanotweezers and nanowires [19]. In some limited studies, microbeams and microplates were modeled by Timoshenko beam theory [17]. The effects of rotary inertia and shear deformation on the nonlinear free vibration of a microbeam were analyzed by Ramezani et al. [20]. Asghari et al. developed governing equations of a microbeam with the assumption of Timoshenko theory, based on the couple stress theory, and analyzed its nonlinear free vibrations [21]. Moghimi Zand and Ahmadian numerically investigated nonlinear vibration of a microplate actuated by electrostatic force with considering the effect of squeeze film damping. They employed the first-order shear deformation theory to model the microplate [11]. For further studies on MEMS/NEMS, please see [22-37].

To solve the nonlinear equations of MEMS, both numerical and semi-analytical methods have been utilized. In the numerical methods, stability and convergence of the solution should be considered because neglecting this issue can result in inappropriate results. Some numerical methods used in this field can be mentioned as Generalized Differential Quadrature method (GDQ) [38], Finite Element method [12], and Shooting method [39]. On the other hand, semi-analytic methods, due to providing a closed-form solution for problems, have been enormously taken into consideration. Among analytical methods, Perturbation method [40] [41] has been mostly used for weak nonlinear problems. The method seeks to find a small parameter and insert it into equation. As a result, finding this parameter is one of its deficiencies [42]. Another analytical method, Homotopy Analysis Method (HAM), which has recently been popular among researchers, is a strong method to solve nonlinear equations. A small parameter is not required in this method. The great trait of this method is controlling sand adjusting of the convergence region. This method was presented by Liao in $1992[43,44]$. In a new study, Daneshpajooh and Moghimi Zand investigated dynamic behavior of an initially curved microbeam using HAM [45].

In the present study, using Lagrange's equations, equations of micro/nano beam dynamics, actuated by electrostatic force, are developed. To obtain the governing equations and relevant boundary conditions, Timoshenko beam theory is considered. Afterwards, using Galerkin's decomposition method, the partial governing equations of motions are converted into nonlinear ordinary differential equations. Hereafter, the Homotopy analysis method is employed to solve the equations and a closed-form solution for each deflection and the frequency of the microbeam is presented. In the next step, some numerical results are presented and the effects of different parameters on the oscillations of the system are investigated. At the end, the applications and summary are presented.

\section{Formulation}

Figure 1 shows an electrostatically actuated microsystem consisting of a clamped-clamped microbeam suspended above a substrate. Through applying voltage, $V$, between the microbeam and substrate, an attractive electrostatic force results in beam deflection. $x, z$, $w$, and $t$ are the coordinate along the length, the coordinate along the thickness, the deflection in the $z$ direction, and time, respectively. $B, \rho$, and $H$ stand for width, density, and thickness of the microbeam, respectively. $\varepsilon, I$, and $d$ represent the vacuum permittivity, the moment of inertia of the cross section about the $y$-axis, and the initial air gap, respectively.

Regarding the Palmer's formula, the electrostatic force per unit area is defined as [16]:

$$
F_{e}=\frac{1}{2} \frac{\varepsilon V^{2}}{(d-w(x, t))^{2}}\left(1+\beta \frac{d-w(x, t)}{b}\right) .
$$

Consider a Timoshenko beam of length $L$, constant cross section of $A$, the mass per unit of $m$, young modulus $E$, and shear modulus $G$ (Figure 1). The displacement field for Timoshenko beam theory is given as follows [46]:

$$
u_{1}=u_{0}(x, t)-z \phi(x, t), \quad u_{2}=0, \quad u_{3}=w(x, t),
$$

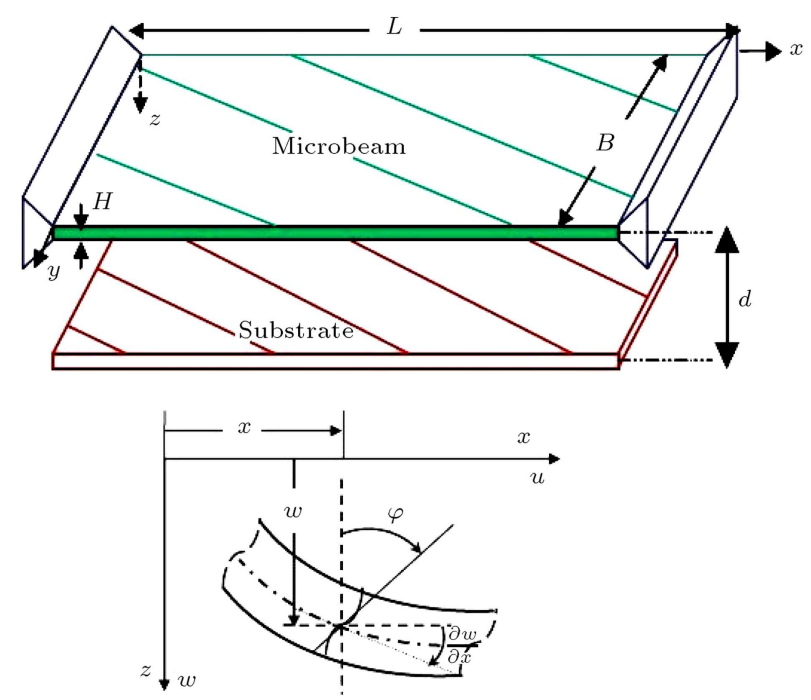

Figure 1. Beam configuration and coordinate system. 
where $u_{1}, u_{2}$, and $u_{3}$ are displacements along $x, y$, and $z$ directions, respectively. $w(x, t), \phi(x, t)$, and $u_{0}(x, t)$, respectively, are the deflection of the beam in $z$ direction, the angle of rotation of cross section about the $y$ axis with respect to the $z$ direction, and the axial displacement of the middle surface. The nonzero components of Strain tensor are defined as follows:

$$
\begin{aligned}
& \varepsilon_{x}=\frac{\partial u}{\partial x}+\frac{1}{2}\left(\frac{\partial w}{\partial x}\right)^{2}, \\
& \gamma_{x z}=\frac{\partial w}{\partial x}-\phi .
\end{aligned}
$$

For the non-zero components of stress tensor, we have:

$$
\begin{aligned}
& \sigma_{x}=E \varepsilon_{x}, \\
& \tau_{x z}=G \gamma_{x z} .
\end{aligned}
$$

In the following, the Lagrange method, which is derived from Hamilton's principle, is employed to develop the governing equations and the relevant boundary conditions. The Kinetic energy and potential energy of a beam are respectively given as follows:

$$
\begin{aligned}
& T=\frac{1}{2} \int_{0}^{L}\left[m_{0}\left(\frac{\partial w}{\partial t}\right)^{2}+m_{0}\left(\frac{\partial u}{\partial t}\right)^{2}+m_{2}\left(\frac{\partial \phi}{\partial t}\right)^{2}\right] d x \\
& U=\frac{1}{2} \int_{0}^{L}\left[E I\left(\frac{\partial \phi}{\partial x}\right)^{2}+K A G \gamma^{2}+E A \varepsilon_{x}^{2}\right] d x
\end{aligned}
$$

where:

$$
\begin{aligned}
& m_{0}=\int_{A} \rho d A=\rho A, \quad I=\int_{A} z^{2} d A, \\
& m_{2}=\int_{A} \rho z^{2} d A=\rho I .
\end{aligned}
$$

In the Lagrange method [47], to consider external forces which are applied to the system, virtual work done by each of the forces is defined based on the virtual displacements of generalized coordinates as follows:

$$
\begin{aligned}
& W_{t}=\vec{F} \cdot \vec{r}, \\
& \overrightarrow{r_{F_{e}}}=\vec{w}(x, t) \hat{k}, \\
& \vec{F}=F_{e} \hat{k},
\end{aligned}
$$

where $W_{t}, \vec{F}, \vec{r}$, and $\overrightarrow{r_{F_{e}}}$ are the work done by total force, the total force vector, the system displacement vector, and the displacements associated with electrostatic force, respectively.

Therefore, virtual work is calculated as follows:

$$
\delta W_{t}=\vec{F} \cdot \overrightarrow{\delta r}=\left(F_{e} \hat{k}\right) \cdot(\overrightarrow{\delta w}(x, t) \hat{k})=F_{e} \delta \vec{w}(x, t) .
$$

The coefficient of each virtual displacement is known as generalized force of the respective coordinate, $Q_{q}$, where $q$ represents the generalized coordinate. Consequently, we have:

$$
\left\{\begin{array}{l}
Q_{w}=F_{e} \\
Q_{\phi}=0 \\
Q_{u}=0
\end{array}\right.
$$

Lagrangian is defined as [48]:

$$
L=T-U \text {. }
$$

And the general form of Lagrange equation is as follows:

$$
\frac{\partial L}{\partial q}-\frac{\partial}{\partial x} \frac{\partial L}{\partial q_{x}}-\frac{\partial}{\partial t} \frac{\partial L}{\partial q_{t}}=-Q_{q}
$$

For each of the generalized coordinates, Eq. (15) is rewritten as follows:

$$
\begin{aligned}
q=w \rightarrow m_{0} \frac{\partial^{2} w}{\partial t^{2}}-\frac{\partial}{\partial x}\left[K A G\left(\frac{\partial w}{\partial x}-\phi\right)\right] \\
-\frac{\partial}{\partial x}\left\{E A\left[\frac{\partial u}{\partial x}+\frac{1}{2}\left(\frac{\partial w}{\partial x}\right)^{2}\right] \frac{\partial w}{\partial x}\right\}=n F_{e}, \quad(16) \\
q=\phi \rightarrow m_{2} \frac{\partial^{2} \phi}{\partial t^{2}}-\frac{\partial}{\partial x}\left(E I \frac{\partial \phi}{\partial x}\right) \\
+K A G\left(\frac{\partial w}{\partial x}-\phi\right)=0, \\
q=u \rightarrow m_{0} \frac{\partial^{2} u}{\partial t^{2}}-\frac{\partial}{\partial x}\left\{E A\left[\frac{\partial u}{\partial x}+\frac{1}{2}\left(\frac{\partial w}{\partial x}\right)^{2}\right]\right\}=0 .
\end{aligned}
$$

Note that longitudinal displacement in beams is trivial; therefore, $m_{0} \frac{\partial^{2} u}{\partial t^{2}}$ is neglected and the governing equations are stated as follows:

$$
\begin{aligned}
& m_{0} \frac{\partial^{2} w}{\partial t^{2}}-K A G\left(\frac{\partial^{2} w}{\partial x^{2}}-\frac{\partial \phi}{\partial x}\right)-N \frac{\partial^{2} w}{\partial x^{2}}=b F_{e} \\
& m_{2} \frac{\partial^{2} \phi}{\partial t^{2}}-E I-K A G\left(\frac{\partial w}{\partial x}-\phi\right)=0
\end{aligned}
$$

where:

$$
N=N_{s}+\frac{E A}{2 L} \int_{0}^{L}\left(\frac{\partial w}{\partial x}\right)^{2} d x,
$$

in which $N_{s}$ is the initial (residual) axial load.

The kinematic boundary conditions and the initial conditions for the microbeam are respectively given as:

$$
\begin{aligned}
& w(0, t)=0, \quad w(L, t)=0, \quad \frac{\partial w}{\partial x}(0, t)=0, \\
& \frac{\partial w}{\partial x}(L, t)=0, \quad \phi(0, t)=0, \quad \phi(L, t)=0,
\end{aligned}
$$




$$
\begin{aligned}
& w(x, 0)=0, \quad \frac{\partial w}{\partial t}(x, 0)=0 \\
& \phi(x, 0)=0, \quad \frac{\partial \phi}{\partial t}(x, 0)=0 .
\end{aligned}
$$

The Taylor's series approximation of $F_{e}$ in Eq. (1) can be written as (the order of approximation is 4$)$ :

$$
\begin{aligned}
F_{e} & =\frac{1}{2} \frac{\varepsilon V^{2}}{(d-w(x, t))^{2}}\left(1+\beta \frac{d-w(x, t)}{B}\right) \\
& =\frac{1}{2} \varepsilon V^{2}\left(\frac{1}{d^{2}}+\frac{2 w(x, t)}{d^{3}}+\frac{3 w(x, t)^{2}}{d^{4}}\right. \\
& \left.+\frac{4 w(x, t)^{3}}{d^{5}}+\frac{5 w(x, t)^{4}}{d^{6}}\right)+\frac{1}{2} \frac{\varepsilon \beta V^{2}}{B}\left(\frac{1}{d}\right. \\
& \left.+\frac{w(x, t)}{d^{2}}+\frac{w(x, t)^{2}}{d^{3}}+\frac{w(x, t)^{3}}{d^{4}}+\frac{w(x, t)^{4}}{d^{5}}\right) .
\end{aligned}
$$

Through combining Eqs. (19a) and (19b) and making some mathematical simplifications, we arrive at the following partial equation which governs the beam deflection:

$$
\begin{aligned}
T(w)= & a_{20} \frac{\partial^{4} w}{\partial x^{4}}+a_{21} \frac{\partial^{4} w}{\partial x^{2} \partial t^{2}}+a_{22} \frac{\partial^{2} w}{\partial t^{2}} \\
& +a_{23} \frac{\partial^{2} w}{\partial x^{2}}+a_{24}\left(\frac{\partial w}{\partial x}\right)^{2}+a_{25} w \frac{\partial^{2} w}{\partial x^{2}} \\
& +a_{26} w\left(\frac{\partial w}{\partial x}\right)^{2}+a_{27} w^{2} \frac{\partial^{2} w}{\partial x^{2}}+a_{28}\left(\frac{\partial w}{\partial t}\right)^{2} \\
& +a_{29} w \frac{\partial^{2} w}{\partial t^{2}}+a_{30} w\left(\frac{\partial w}{\partial t}\right)^{2}+a_{31} w^{2} \frac{\partial^{2} w}{\partial t^{2}} \\
& +a_{32} \frac{\partial^{4} w}{\partial t^{4}}+a_{33} w+a_{34} w^{2}+a_{35} w^{3}+a_{36} \\
& +a_{30} w^{2}\left(\frac{\partial w}{\partial x}\right)^{2}+a_{38} w^{3} \frac{\partial^{2} w}{\partial x^{2}} \\
& +a_{39} w^{2}\left(\frac{\partial w}{\partial t}\right)^{2}+a_{40} w^{3} \frac{\partial^{2} w}{\partial t^{2}}+a_{41} w^{4}=0
\end{aligned}
$$

Coefficients $a_{20}-a_{41}$ are presented in Appendix A. For more realistic results for nano systems, one may want to consider intermolecular forces.

To obtain the governing equation and the boundary conditions in the dimensionless form, the following variables are introduced:

$$
\hat{x}=\frac{x}{L} \hat{w}=\frac{w}{d} \hat{t}=\frac{t}{t^{*}},
$$

where:

$$
t^{*}=\sqrt{\frac{m L^{4}}{E I}} .
$$

Using Eq. (25), the governing equation is rewritten as:

$$
\begin{aligned}
T(\hat{w})= & \frac{\partial^{4} \hat{w}}{\partial \hat{x}^{4}}+\frac{\partial^{2} \hat{w}}{\partial \hat{t}^{2}}+b_{1} \frac{\partial^{4} \hat{w}}{\partial \hat{x}^{2} \partial \hat{t}^{2}}+b_{2} \frac{\partial^{2} \hat{w}}{\partial \hat{t}^{2}}+b_{3} \frac{\partial^{2} \hat{w}}{\partial \hat{x}^{2}} \\
& +b_{4} \frac{\partial^{4} \hat{w}}{\partial \hat{x}^{4}}+b_{5} \frac{\partial^{4} \hat{w}}{\partial \hat{x}^{2} \partial \hat{t}^{2}}+b_{6} \frac{\partial^{2} \hat{w}}{\partial \hat{x}^{2}}+b_{7}\left(\frac{\partial \hat{w}}{\partial \hat{x}}\right)^{2} \\
& +b_{8} \hat{w} \frac{\partial^{2} \hat{w}}{\partial \hat{x}^{2}}+b_{9} \hat{w}\left(\frac{\partial \hat{w}}{\partial \hat{x}}\right)^{2}+b_{10} \hat{w}^{2} \frac{\partial^{2} \hat{w}}{\partial \hat{x}^{2}} \\
& +b_{11} \hat{w}^{2}\left(\frac{\partial \hat{w}}{\partial \hat{x}}\right)^{2}+b_{12} \hat{w}^{3} \frac{\partial^{2} \hat{w}}{\partial \hat{x}^{2}}+b_{13} \hat{w} \\
& +b_{14} \hat{w}^{2}+b_{15} \hat{w}^{3}+b_{16} \hat{w}^{4}+b_{17}+b_{18} \frac{\partial^{4} \hat{w}}{\partial \hat{t}^{4}} \\
& +b_{19}\left(\frac{\partial \hat{w}}{\partial \hat{t}}\right)^{2}+b_{120} \hat{w} \frac{\partial^{2} \hat{w}}{\partial \hat{t}^{2}}+b_{21} \hat{w}\left(\frac{\partial \hat{w}}{\partial \hat{t}}\right)^{2} \\
& +b_{22} \hat{w}^{2} \frac{\partial^{2} \hat{w}}{\partial \hat{t}^{2}}+b_{23} \hat{w}^{2}\left(\frac{\partial \hat{w}}{\partial \hat{t}}\right)^{2} \\
& +b_{24} \hat{w}^{3} \frac{\partial^{2} \hat{w}}{\partial \hat{t}^{2}}=0 .
\end{aligned}
$$

Coefficients $b_{1}-b_{24}$ are presented in Appendix B. The dimensionless Kinematic boundary conditions and initial conditions are defined as:

$$
\begin{aligned}
& \hat{w}(0, \hat{t})=0 \hat{w}(1, \hat{t})=0 \frac{\partial \hat{w}}{\partial \hat{x}}(0, \hat{t})=0 \frac{\partial \hat{w}}{\partial \hat{x}}(1, \hat{t})=0 \\
& \hat{w}(\hat{x}, 0)=0 \hat{w}(\hat{x}, 1)=0 .
\end{aligned}
$$

In order to solve Eq. (27), the deflection of the beam is assumed to be the product of two separated functions as follows:

$$
\hat{w}(\hat{x}, \hat{t})=\varphi(\hat{x}) u(\hat{t}),
$$

where $\varphi(\hat{x})$ is a trial function which satisfies the kinematic boundary conditions and $u(\hat{t})$ is an unknown time-dependent function. $\varphi(\hat{x})$ can be defined as:

$$
\varphi(\hat{x})=\hat{x}^{2}(1-\hat{x})^{2} .
$$

The one-parameter Galerkin's solution can be computed by:

$$
\int_{0}^{1} \varphi(\hat{x}) T(\hat{w}(\hat{x}, \hat{t})) d x=0 .
$$

After substituting Eqs. (27) and (31) in Eq. (32) and 
integrating them, the governing equation for $u(\hat{t})$ is obtained as follows:

$$
\begin{aligned}
u^{(4)} & +\left(a_{1}+a_{13} V^{2}\right) \ddot{u}+\left(a_{2}+a_{14} V^{2}\right) u+a_{3} V^{2} u^{2} \\
& +\left(a_{4}+a_{15} V^{2}\right) u^{3}+a_{5} V^{2} u^{4}+a_{6} V^{2} \dot{u}^{2} \\
& +a_{7} V^{2} u \dot{u}^{2}+a_{8} V^{2} u^{2} \dot{u}^{2}+a_{9} V^{2} u \ddot{u}+\left(a_{10}\right. \\
& \left.+a_{16} V^{2}\right) u^{2} \ddot{u}+a_{11} V^{2} u^{3} \ddot{u}+a_{12} V^{2}=0 .
\end{aligned}
$$

$a_{1}-a_{16}$ are given in Appendix C. The initial conditions are mentioned as:

$$
u(0)=0, \dot{u}(0)=0, \ddot{u}(0)=0, u^{(3)}(0)=0 .
$$

In the next section, the homotopy analysis method is employed to solve Eq. (33).

\section{Application of homotopy analysis method to the problem}

In this section, initially, a brief description of the homotopy analysis method is presented.

HAM transforms a general nonlinear problem into an infinite number of linear problems by embedding an auxiliary parameter $q$. Consider the following nonlinear differential equation:

$$
\mathcal{R}(u(t))=0,
$$

where $\mathcal{R}$ is a nonlinear operator and $u(t)$ is an unknown function. Using $q \in[0,1]$ as an embedding parameter, the defined homotopy is introduced as follows [44]:

$$
\begin{gathered}
\mathcal{H}(\Phi ; q ; h, \quad H(\hat{t}))=(1-q) \mathcal{L}\left[\Phi(\hat{t} ; q)-u_{0}(\hat{t})\right] \\
-q h H(\hat{t}) \mathcal{R}\left[\Phi(\hat{t} ; q), f_{t}(q), h_{t}(q)\right],
\end{gathered}
$$

where $u_{0}(t), H(\hat{t}), \mathcal{L}$, and $\mathcal{R}$ stand for non-zero auxiliary parameter, an initial guess, non-zero auxiliary function, auxiliary linear operator, and nonlinear operator, respectively. Values of $h$ and $H(\hat{t})$ adjust the convergence region of the solution. For a microbeam problem, the auxiliary function is 1 .

Considering Eq. (33), the nonlinear operator may be written as:

$$
\begin{aligned}
\mathcal{R}[\Phi(t ; q), & \left.f_{t}(q), h_{t}(q)\right]=\frac{\partial^{4} \Phi(t ; q)}{\partial t^{4}}+\left(f_{t}(q)\right. \\
& \left.+h_{t}(q)\right) \frac{\partial^{2} \Phi(t ; q)}{\partial t^{2}}+f_{t}(q) h_{t}(q) \Phi(t ; q) \\
& +a_{3} V^{2} \Phi(t ; q)^{2}+\left(a_{4}+a_{15} V^{2}\right) \Phi(t ; q)^{3} \\
& +a_{5} V^{2} \Phi(t ; q)^{4}+a_{6} V^{2}\left(\frac{\partial \Phi(t ; q)}{\partial t}\right)^{2}
\end{aligned}
$$

$$
\begin{aligned}
& +a_{7} V^{2} \Phi(t ; q)\left(\frac{\partial \Phi(t ; q)}{\partial t}\right)^{2}+a_{8} V^{2} \Phi(t ; q)^{2} \\
& \left(\frac{\partial \Phi(t ; q)}{\partial t}\right)^{2}+a_{9} V^{2} \Phi(t ; q) \frac{\partial^{2} \Phi(t ; q)}{\partial t^{2}} \\
& +\left(a_{10}+a_{16} V^{2}\right) \Phi(t ; q)^{2} \frac{\partial^{2} \Phi(t ; q)}{\partial t^{2}} \\
& +a_{11} V^{2} \Phi(t ; q)^{3} \frac{\partial^{2} \Phi(t ; q)}{\partial t^{2}}+a_{12} V^{2}
\end{aligned}
$$

Note that:

$$
\begin{aligned}
& f_{t}(1)=\frac{\left(a_{1}+a_{13} V^{2}\right)}{2} \sqrt{\frac{\left(a_{1}+a_{13} V^{2}\right)^{2}}{4}-\left(a_{2}+a_{14} V^{2}\right)}, \\
& h_{t}(1)=\frac{\left(a_{1}+a_{13} V^{2}\right)}{2} \sqrt{\frac{\left(a_{1}+a_{13} V^{2}\right)^{2}}{4}-\left(a_{2}+a_{14} V^{2}\right)} .
\end{aligned}
$$

The homotopy linear operator is assumed as:

$$
\begin{aligned}
\mathcal{L}[\Phi(t ; q)]= & \frac{\partial^{4} \Phi(t ; q)}{\partial t^{4}}+\left(\omega^{2}+v^{2}\right) \frac{\partial^{2} \Phi(t ; q)}{\partial t^{2}} \\
& +\omega^{2} v^{2} \Phi(t ; q),
\end{aligned}
$$

in which $\omega$ and $\nu$ are the bending and the rotary natural frequencies, respectively. Note that the bending natural frequency is only investigated in this study. The functions $\Phi(t ; q), f_{t}(q)$, and $h_{t}(q)$ can be expanded as:

$$
\begin{gathered}
f_{t}(q)=\omega^{2}+\omega_{1}(\omega) q+\omega_{2}(\omega) q^{2}+\omega_{3}(\omega) q^{3}+\omega_{4}(\omega) q^{4}+\ldots \\
h_{t}(q)=v^{2}+v_{1}(v) q+v_{2}(v) q^{2}+v_{3}(v) q^{3}+v_{4}(v) q^{4}+\ldots \\
\Phi(t ; q)=u_{0}(t)+u_{1}(t) q+u_{2}(t) q^{2}+u_{3}(t) q^{3} \\
\quad+u_{4}(t) q^{4}+u_{5}(t) q^{5}+u_{6}(t) q^{6}+\ldots
\end{gathered}
$$

By equating Eq. (36) with zero, the zero-order deformation equation is obtained as:

$$
\begin{aligned}
& (1-q) \mathcal{L}\left[\Phi(\hat{t} ; q)-u_{0}(\hat{t})\right]=q h \mathcal{R}\left[\Phi(\hat{t} ; q), f_{t}(q), h_{t}(q)\right] \\
& \Phi(0 ; q)=0, \quad \frac{\partial \Phi(0 ; q)}{\partial t}=0 \\
& \frac{\partial^{2} \Phi(0 ; q)}{\partial t^{2}}=0, \quad \frac{\partial^{3} \Phi(0 ; q)}{\partial t^{3}}=0
\end{aligned}
$$

when $q=0$, we arrive at:

$$
\mathcal{L}\left[\Phi(\hat{t} ; q)-u_{0}(\hat{t})\right]=0 .
$$

Therefore, the zero-order approximation of $u(t)$ can be calculated. 
$u_{o}(\hat{t})$ can be set to zero. In the next step, with differentiating Eq. (43) and then setting $q=0$, the first-order deformation equation is constructed. Through solving the following equation, the first-order approximation of $u(t)$ is obtained subject to zero initial conditions:

$$
\mathcal{L}\left[u_{1}(t)\right]=\left.h \mathcal{R}\left[\Phi(t ; q), f_{t}(q), h_{t}(q)\right]\right|_{q=0} .
$$

The higher-order approximations of the solution $u(t)$ can be obtained by solving high-order deformation equations. Differentiating Eq. (43) $j$ times with respect to $q$, then setting $q=0$, and finally dividing each side by $j$ !, the $j$ th order deformation equation is obtained as follows:

$$
\begin{aligned}
& \mathcal{L}\left[u_{j}(t)-\chi_{j} u_{j-1}(t)\right]=\frac{1}{(j-1) !} \\
& \left.h \frac{\partial^{j-1} \mathcal{R}\left[\Phi(t ; q), f_{t}(q), h_{t}(q)\right]}{\partial q^{j-1}}\right|_{q=0},
\end{aligned}
$$

in which $\chi_{j}$ is defined:

$$
\chi_{j}= \begin{cases}0 & \text { when } j \leq 1 \\ 1 & \text { otherwise }\end{cases}
$$

The terms $\omega_{j}$ and $v_{j}$ are obtained through eliminating the secular terms. It should be noted that vibrations of an undamped microbeam under the actuation of the electrostatic force can be expressed by the following base functions [16]:

$$
\cos (k \omega t), \cos (k v t) k=1,2,3, \ldots
$$

Therefore, to eliminate the secular term in the $j$ th order of approximation, the coefficients of $\cos (k \omega t)$ and $\cos (k v t)$ in the $(j-1)$ th order deformation equation have to be set to zero. This results in two algebraic equations. Solving these two equations yields $\omega_{j-2}$ and $v_{j-2}$ as functions of both $\omega$ and $v$. After obtaining sufficient approximations, by setting $q=1$ in Eqs. (40), (41), and (42), we arrive at:

$$
\begin{aligned}
f_{t}(1) & =\frac{\left(a_{1}+a_{13} V^{2}\right)}{2}-\sqrt{\frac{\left(a_{1}+a_{13} V^{2}\right)^{2}}{4}-\left(a_{2}+a_{14} V^{2}\right)} \\
& =\omega^{2}+\sum_{j=1}^{p} \omega_{j}(\omega)=\omega^{2}+\omega_{1}(\omega)+\omega_{2}(\omega)+\ldots+\omega_{p}(\omega), \\
h_{t}(1) & =\frac{\left(a_{1}+a_{13} V^{2}\right)}{2}+\sqrt{\frac{\left(a_{1}+a_{13} V^{2}\right)^{2}}{4}-\left(a_{2}+a_{14} V^{2}\right)} \\
& =v^{2}+\sum_{j=1}^{p} v_{j}(v)=v^{2}+v_{1}(v)+v_{2}(v)+\ldots+v_{p}(v),
\end{aligned}
$$

$$
\begin{aligned}
u(t)= & \sum_{j=1}^{p+2} u_{j}(t)=u_{0}(t)+u_{1}(t)+u_{2}(t)+u_{3}(t) \\
& +\ldots+u_{p+2}(t)
\end{aligned}
$$

where $p$ is order of approximation. The nonlinear frequencies $\omega$ and $v$ can be calculated through simultaneously solving Eqs. (50) and (51), using MATLAB fsolve command. The terms $u_{1}(t), u_{2}(t), \omega_{1}, \omega_{2}, v_{1}$, and $v_{2}$ are presented in Appendix D.

\section{Results and discussion}

In this section, some numerical examples are presented to investigate the behavior of a microbeam, actuated by electrostatic force, with general assumption of Timoshenko beam theory. Also, results are compared with those obtained based on the assumption of EulerBernoulli beam theory, which is available in literature.

In the first step, in order to validate the model used in this study, some comparisons are made between the present model and the model developed with assumption of Euler-Bernoulli beam theory (available in literature). Moreover, the results obtained by the present model are compared with numerical results. Figure 2(a) and (b) compare the results obtained by the present model with those presented in literature (with assumption of Euler-Bernoulli beam theory). Figure 2(a) and (b) display the variations of the nonlinear frequency with applied voltage and the midpoint deflection time history, respectively. Note that to draw these figures, the parameters are selected as [16]: $B=100 \mu \mathrm{m}, H=1.5 \mu \mathrm{m}, \rho=2330 \frac{\mathrm{kg}}{\mathrm{m}^{3}}, v=0.28$, $d=1.18 \mu \mathrm{m}, E=166 \mathrm{GPa}$, and $R S=6 \mathrm{MPa}$. Results provided in Figure 2(a) and (b) show that in slender beams, results of Euler-Bernoulli and Timoshenko beam theories are in close agreement, which are confirmed by the results available in literature [49].

Table 1 presents the calculated [38] and measured [50] initial frequencies for a microbeam in different beam lengths. The results provided in Table 1 also confirm that in beams with the large $\frac{L}{H}$ ratio, Timoshenko and Euler-Bernoulli beam theories are in good agreement.

Figure 3 illustrates the midpoint deflection of a microbeam with $L=150 \mu \mathrm{m}, B=50 \mu \mathrm{m}, d=$ $2 \mu \mathrm{m}, H=7 \mu \mathrm{m}, \rho=2230 \frac{\mathrm{Kg}}{\mathrm{m}^{3}}, v=0.28, E=$ $169 \mathrm{GPa}, V=100 \mathrm{~V}$, and $R S=0 \mathrm{MPa}$. The beam equation is numerically solved by Runge-Kutta method, using MATLAB software, and the numerical results are presented in Figure 3 . It is seen that there exists an excellent agreement between the analytical and numerical results.

The comparisons reveal that the model utilized in this study is reliable. In order to show the difference between Euler-Bernoulli and Timoshenko beam 


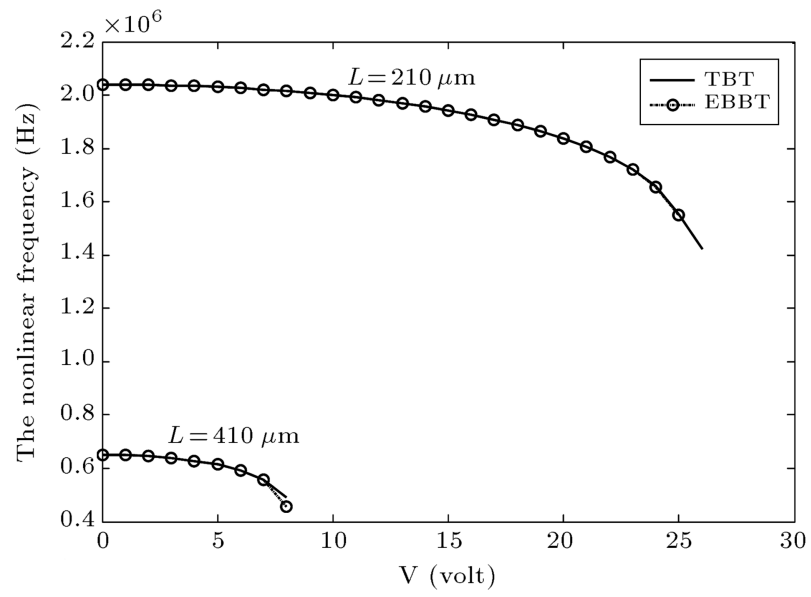

(a)

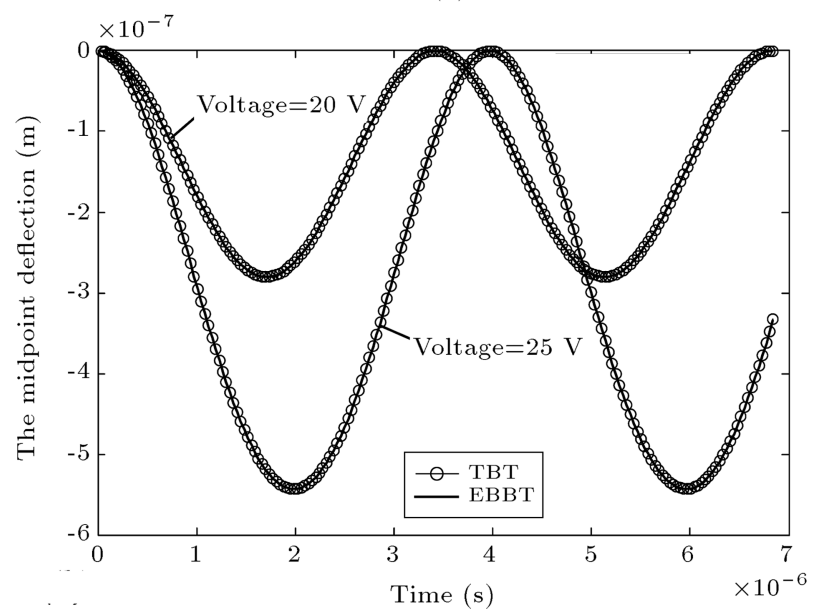

(b)

Figure 2. Comparison between the predictions of Euler-Bernoulli and Timoshenko beam theories about: (a) the variations of the nonlinear frequency $(\mathrm{Hz})$ with applied voltage (V) for different beam lengths, and (b) the midpoint deflection time history for different input voltages and $L=210 \mu \mathrm{m}$.

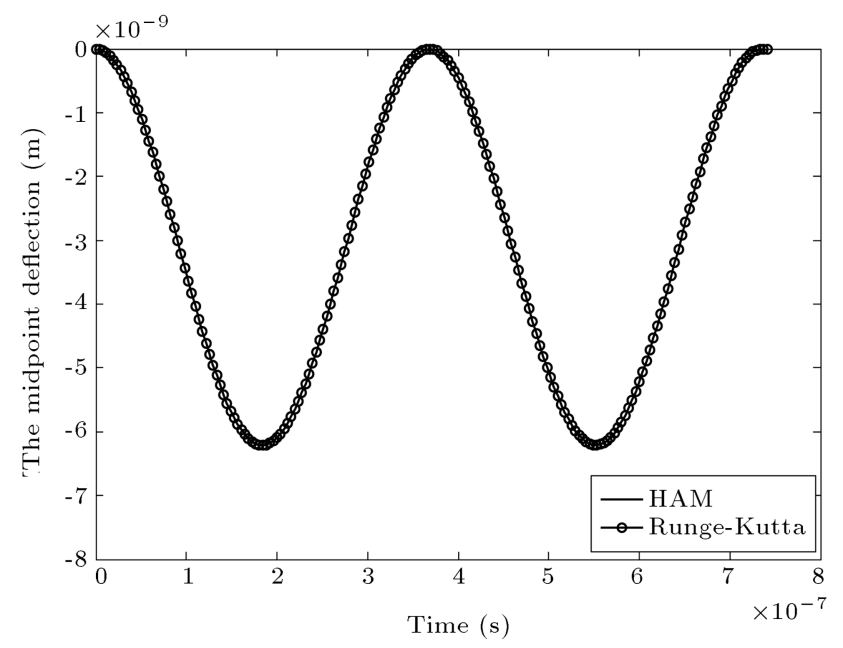

Figure 3. Comparison between the results (the midpoint deflection time history) obtained by the Homotopy Analysis Method (HAM) and Runge-Kutta Method. theories, Figures 4 and 5, respectively, display the variations of the nonlinear frequency with the applied voltage and the midpoint deflection time history of a microbeam. Note that Figure 5(a)-(c) illustrate the midpoint deflection time history for the input voltages near the voltage of pull-in instability. The parameters are selected as: $L=550 \mu \mathrm{m}, B=50 \mu \mathrm{m}, \rho=2230 \frac{\mathrm{Kg}}{\mathrm{m}^{3}}$, $v=0.28, d=1 \mu \mathrm{m}, E=169 \mathrm{GPa}$, and $R S=0 \mathrm{MPa}$. It is observed that with increasing the input voltage or the beam thickness, the predictions of Timoshenko beam theory differ from the predictions of EulerBernoulli beam theory. Table 2 also provides some nonlinear frequency values for an actuated microbeam with parameters used in Figure 4, for different input voltages and beam thicknesses.

Table 3 lists the voltage of pull-in instability corresponding to Figures 4 and 5 . The results presented in this table also confirm that in thick beams, the differences between the above-mentioned theories are obvious. The effect of residual stress on the nonlinear frequency and the midpoint deflection of a microbeam are depicted in Figure 6(a) and (b). The parameters are: $L=550 \mu \mathrm{m}, B=50 \mu \mathrm{m}, \rho=2230 \frac{\mathrm{Kg}}{\mathrm{m}^{3}}$, $v=0.28, d=1 \mu \mathrm{m}$, and $H=30 \mu \mathrm{m}$. Figure $6(\mathrm{~b})$ depicts deflections for input voltage of $230 \mathrm{~V}$. It is seen that increasing the tensile pretension results in increase in nonlinear frequency. By contrast, increasing the compressive pretension leads to decrease in the nonlinear frequency. This trend is reversed for the midpoint beam defection.

Figure $7(\mathrm{a})$ and (b) present the phase portrait for a microbeam with assumptions of Euler-Bernoulli and Timoshenko beam theories, respectively. The parameters are selected as: $L=550 \mu \mathrm{m}, B=50 \mu \mathrm{m}$, $d=1 \mu \mathrm{m}, H=30 \mu \mathrm{m}, \rho=2230 \frac{\mathrm{Kg}}{\mathrm{m}^{3}}, v=0.28$, $E=169 \mathrm{GPa}$ and $V 230 \mathrm{~V}$.

Figure 8 compares the behavior of a microbeam based on Timoshenko beam theory with that of a microplate based on nonlinear first-order shear deformation theory (FSDT) [51] in different pretension values. Both of them are actuated with the input voltage of $30 \mathrm{~V}$. The parameters in this figure are: $L=250 \mu \mathrm{m}, B=50 \mu \mathrm{m}, d=2 \mu \mathrm{m}, H=2 \mu \mathrm{m}$, and $E=169 \mathrm{GPa}$. It can be observed that the differences between the results obtained for the microbeam and those of the microplate increase as the compressive pretention increases. In HAM, an appropriate value for $h$ parameter has to be found to guarantee the convergence of the solution series. One of the best ways to find this value is to plot $h$-curves. $h$-curves show the variations of solution with respect to $h$. Note that the proper solution should be independent from $h$. Figure 9 depicts the nonlinear frequency versus $h$ in different orders of approximation $p$. It is seen that by setting $h=-1$, we ensure converge of the solution series. 
Table 1. Comparison between the experimental and calculated results.

\begin{tabular}{cccccc}
\hline & \multicolumn{5}{c}{$\frac{\omega_{\mathbf{0}}}{\mathbf{2 \pi}} \mathbf{( \mathbf { k H z } )}$} \\
\cline { 2 - 6 } Beam length $(\boldsymbol{\mu m})$ & $\begin{array}{c}\text { Measured } \\
{[\mathbf{2 4}]}\end{array}$ & $\begin{array}{c}\text { Calculated } \\
{[\mathbf{2 4}]}\end{array}$ & $\begin{array}{c}\text { Calculated } \\
{[\mathbf{1 1}]}\end{array}$ & $\begin{array}{c}\text { Semi-analytic } \\
\text { method for } \\
\text { Euler-Bernoulli } \\
\text { beam [16] }\end{array}$ & $\begin{array}{c}\text { Semi-analytic } \\
\text { Timothod for } \\
\text { menko beam } \\
\text { (present study) }\end{array}$ \\
\hline 210 & 322.05 & 324.70 & 324.70 & 324.78 & 324.69 \\
310 & 163.22 & 164.35 & 163.46 & 163.16 & 163.13 \\
410 & 102.17 & 103.80 & 103.70 & 103.42 & 103.40 \\
510 & 73.79 & 74.80 & 73.46 & 74.38 & 74.36 \\
\hline
\end{tabular}

Table 2. Comparison between the predictions of Euler-Bernoulli and Timoshenko beam theories for the nonlinear frequency of a microbeam in different input voltages and beam thicknesses.

\begin{tabular}{|c|c|c|c|c|c|c|c|c|c|}
\hline $\mathbf{H}(\mu \mathrm{m})$ & \multicolumn{3}{|c|}{10} & \multicolumn{3}{|c|}{20} & \multicolumn{3}{|c|}{30} \\
\hline$V(\mathrm{v})$ & 10 & 25 & 42 & 50 & 100 & 121 & 50 & 200 & 223 \\
\hline TBT & 288.54 & 278.04 & 228.06 & 568.02 & 518.04 & 431.46 & 861.34 & 742.45 & 628.18 \\
\hline EBBT & 288.70 & 278.20 & 230.77 & 569.61 & 519.80 & 446.74 & 866.43 & 749.30 & 665.26 \\
\hline
\end{tabular}
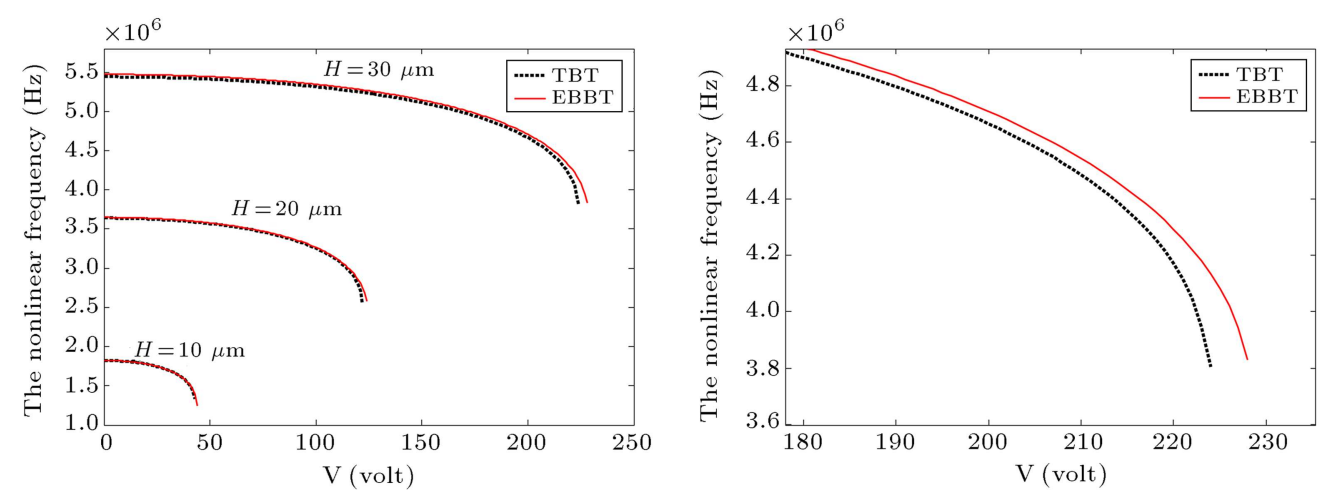

(a)

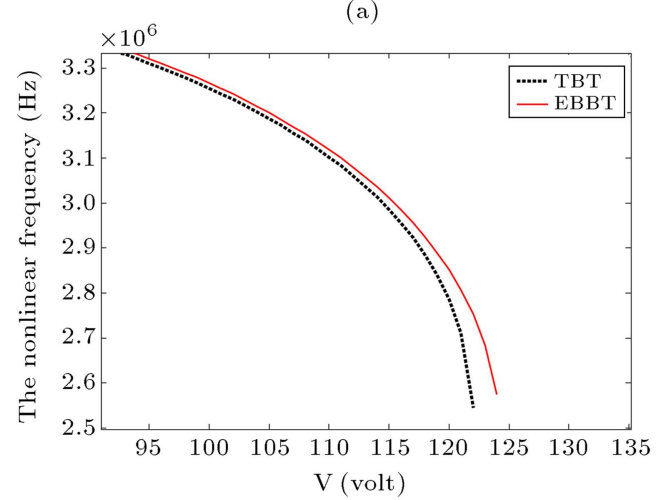

(c)

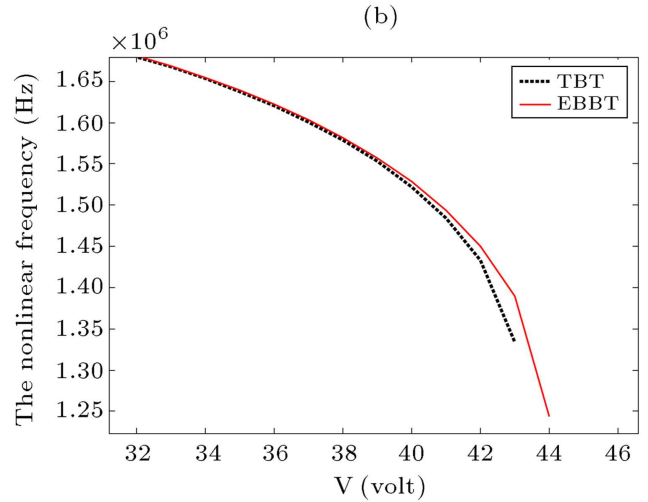

(d)

Figure 4. Comparison between the predictions of Euler-Bernoulli beam theory about the variations of the nonlinear frequency with the input voltage for: (a) Different thicknesses of the beam; (b) the magnification of $H=30 \mu \mathrm{m}$; (c) the magnification of $H=20 \mu \mathrm{m}$; and (d) the magnification of $H=10 \mu \mathrm{m}$.

Table 3. Comparison between predictions of

Euler-Bernoulli and Timoshenko beam theories for the voltage of pull-in instability of an actuated microbeam in different beam thicknesses.

\begin{tabular}{cccc}
\hline $\mathbf{H}(\boldsymbol{\mu m})$ & $\mathbf{1 0}$ & $\mathbf{2 0}$ & $\mathbf{3 0}$ \\
\hline TBT & $46.33 \mathrm{~V}$ & $130.87 \mathrm{~V}$ & $240.06 \mathrm{~V}$ \\
EBBT & $46.34 \mathrm{~V}$ & $131 \mathrm{~V}$ & $240.63 \mathrm{~V}$ \\
\hline
\end{tabular}

\section{Conclusions}

In this study, the governing equations and the boundary conditions of nonlinear oscillation of micro/nano beams with assumption of Timoshenko beam theory have been developed. The ordinary equation of motion has been built utilizing the 


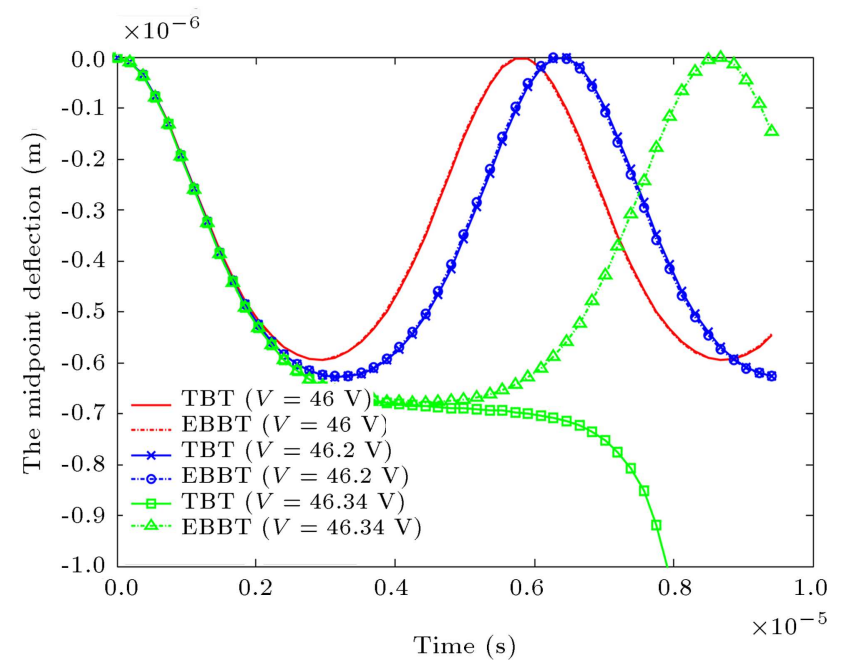

(a)

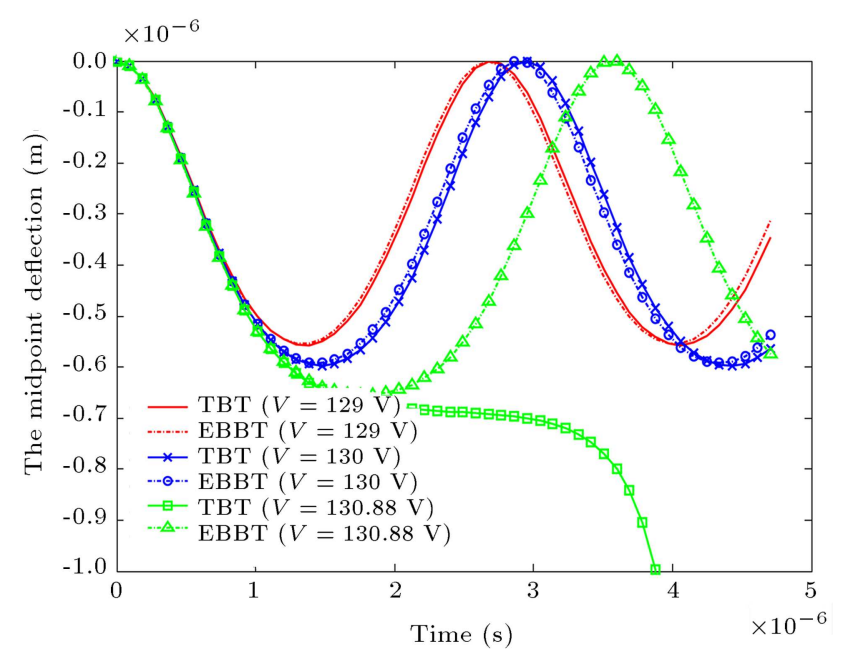

(b)

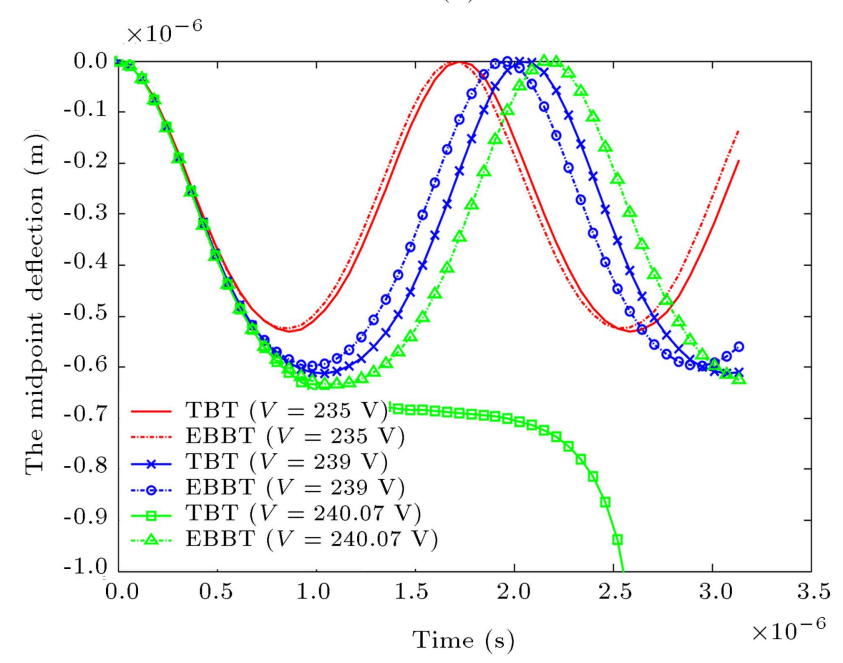

(c)

Figure 5. Comparison between the predictions of Euler-Bernoulli and Timoshenko beam theories about the midpoint deflection time history near the voltage of pull-in instability with beam thicknesses of (a) $H=10 \mu \mathrm{m}$, (b) $H=20 \mu \mathrm{m}$, and (c) $H=30 \mu \mathrm{m}$.

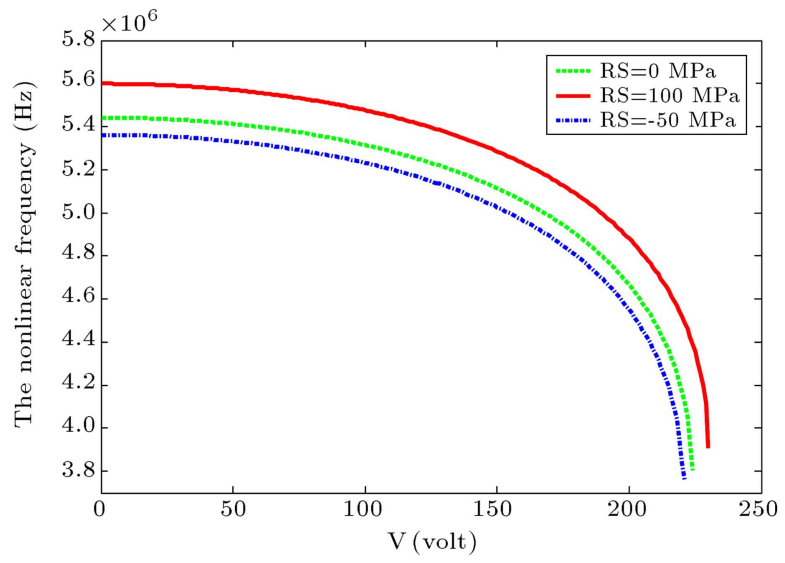

(a)

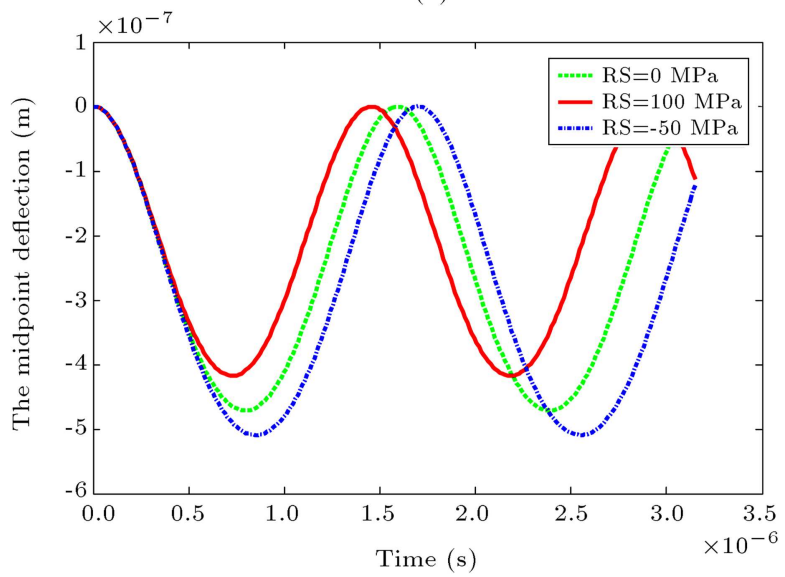

(b)

Figure 6. The effect of residual stress on the (a) variations of the nonlinear frequency with the input voltage, and (b) midpoint deflection time history (voltage $=230 \mathrm{~V})$.

Galerkin's decomposition method. The homotopy analysis method has been employed to solve the governing equation and the present semi-analytical solutions. The results obtained based on the present model have been compared with those obtained by Euler-Bernoulli beam theory. Regarding the presented results for thick beams, Timoshenko beam theory gives us more accurate results, which are in close agreement with experimental results. Furthermore, with increasing the input voltage, the differences between results of Timoshenko and Euler-Bernoulli beam theories increase. Also, comparing the results predicted by FSDT plate and Timoshenko beam, one can note that in low amounts of compressive pretension, these two theories provide approximately the same results. Conversely, in high amounts of compressive pretension, these two theories differ from each other.

\section{Acknowledgments}

The authors would like to thank Iranian National Science Foundation (INSF) for their support. 


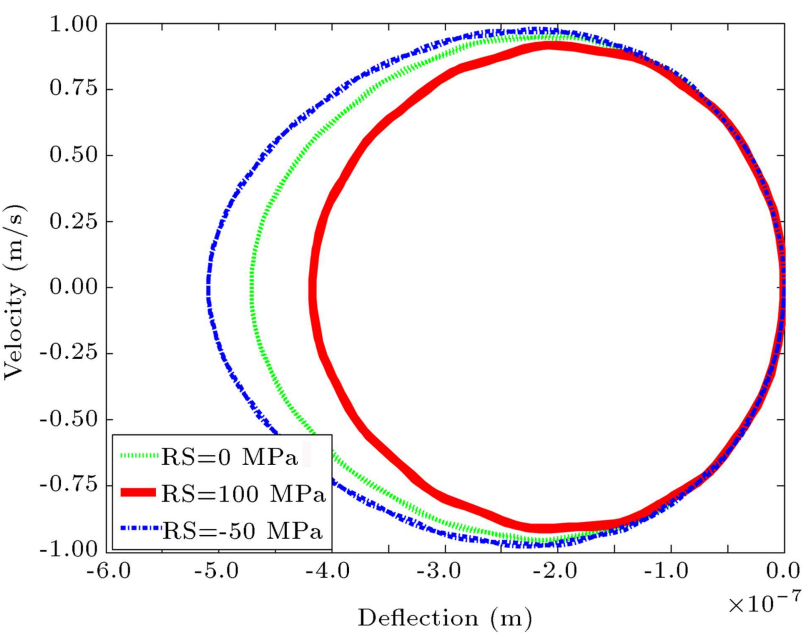

(a)

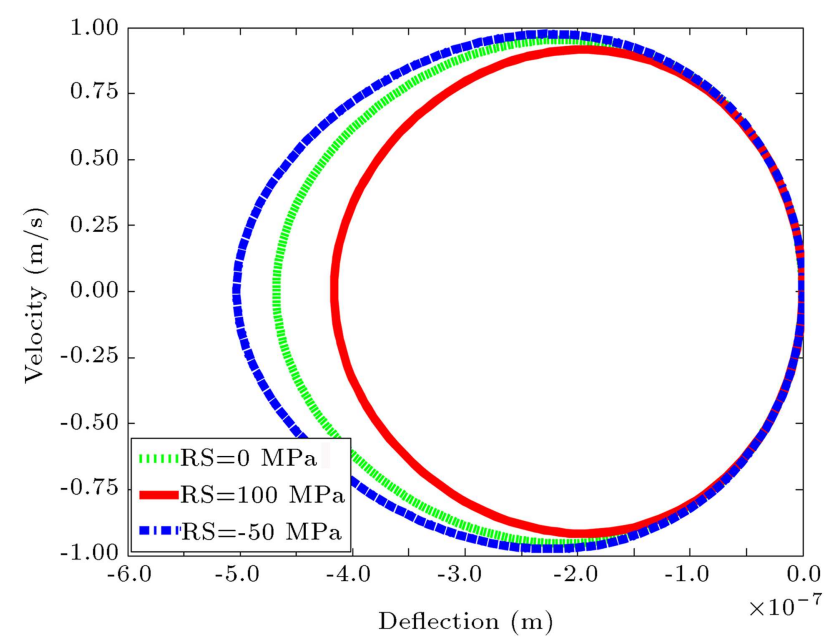

(b)

Figure 7. The phase portrait of a microbeam (voltage $=$ $230 \mathrm{~V}$ ) with assumptions of (a) Euler-Bernoulli theory, and (b) Timoshenko theory.

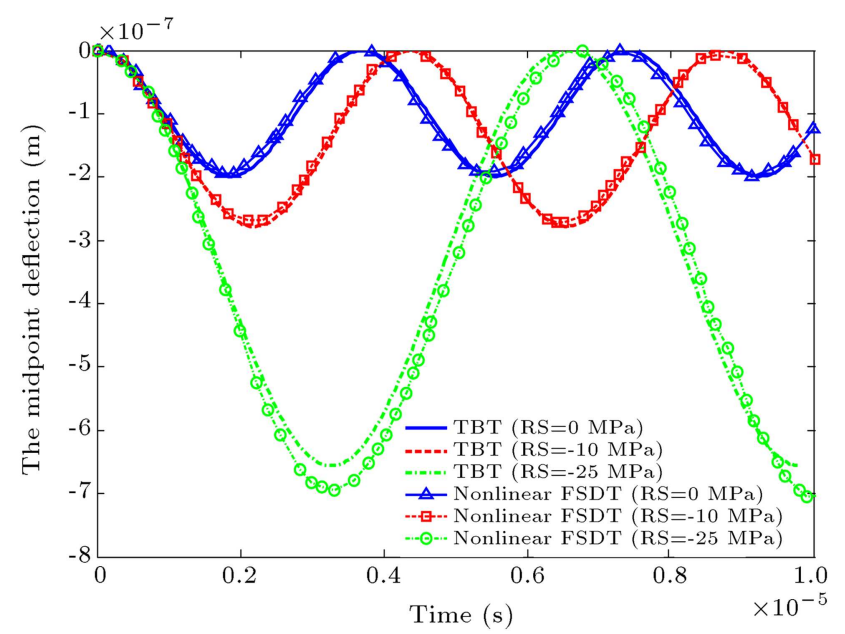

Figure 8. Comparison between a microbeam (with assumption of Timoshenko theory) and a microplate (with assumption of first-shear deformation theory) under different pretension values with the input voltage of $30 \mathrm{~V}$.

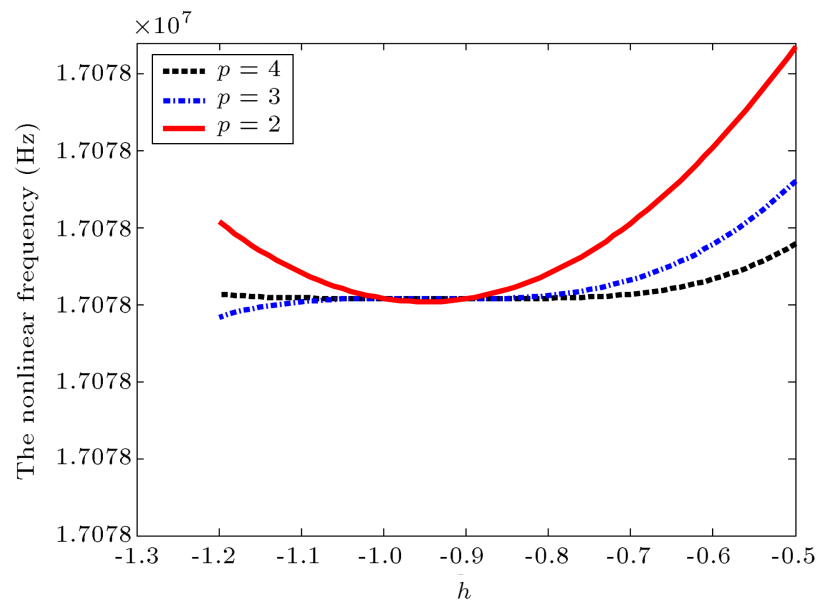

Figure 9. The $h$-curve of $\omega$ for the microbeam actuated by step-input voltage of $100 \mathrm{~V}$.

\section{References}

1. Nathanson, H.C., Newell, W.E., Wickstrom, R., et al. "The resonant gate transistor", Electron Devices, IEEE Trans., 14(3), pp. 117-133 (1967).

2. Taylor, G. "The coalescence of closely spaced drops when they are at different electric potentials", in Proceedings of the Royal Society of London A: Mathematical, Physical and Engineering Sciences, 306(1487), pp. 423-434 (1968).

3. Batra, R.C., Porfiri, M. and Spinello, D. "Vibrations of narrow microbeams predeformed by an electric field", J. Sound Vib., 309(3), pp. 600-612 (2008).

4. Rochus, V., Rixen, D.J. and Golinval, J.-C. "Electrostatic coupling of MEMS structures: transient simulations and dynamic pull-in", Nonlinear Anal. Theory, Methods Appl., 63(5), pp. e1619-e1633 (2005).

5. Krylov, S. "Lyapunov exponents as a criterion for the dynamic pull-in instability of electrostatically actuated microstructures", Int. J. Non. Linear. Mech., 42(4), pp. 626-642 (2007).

6. Zand, M.M. "The dynamic pull-in instability and snapthrough behavior of initially curved microbeams", Mech. Adv. Mater. Struct., 19(6), pp. 485-491 (2012).

7. Mojahedi, M., Zand, M.M., Ahmadian, M.T. and Babaei, M. "Analytic solutions to the oscillatory behavior and primary resonance of electrostatically actuated microbridges", Int. J. Struct. Stab. Dyn., 11(6), pp. 1119-1137 (2011).

8. Zand, M.M. and Ahmadian, M.T. "Dynamic pull-in instability of electrostatically actuated beams incorporating Casimir and van der Waals forces", Proc. Inst. Mech. Eng. Part C J. Mech. Eng. Sci, 224(9), pp. 2037-2047 (2010).

9. Zand, M.M., Rashidian, B. and Ahmadian, M.T. "Contact time study of electrostatically actuated microsystems", Sci. Iran. Trans. B Mech. Engng, 17(5), pp. 348-357 (2010). 
10. Mojahedi, M., Moghimi Zand, M. and Ahmadian, M.T. "Static pull-in analysis of electrostatically actuated microbeams using homotopy perturbation method", Appl. Math. Model., 34(4), pp. 1032-1041 (2010).

11. Moghimi Zand, M. and Ahmadian, M.T. "Vibrational analysis of electrostatically actuated microstructures considering nonlinear effects", Commun. Nonlinear Sci. Numer. Simul., 14(4), pp. 1664-1678 (2009).

12. Moghimi Zand, M. and Ahmadian, M.T. "Characterization of coupled-domain multi-layer microplates in pull-in phenomenon, vibrations and dynamics", Int. $J$. Mech. Sci., 49(11), pp. 1226-1237 (2007).

13. Nayfeh, A.H., Younis, M.I. and Abdel-Rahman, E.M. "Dynamic pull-in phenomenon in MEMS resonators", Nonlinear Dyn., 48(1), pp. 153-163 (2007).

14. Batra, R.C., Porfiri, M. and Spinello, D. "Effects of van der Waals force and thermal stresses on pullin instability of clamped rectangular microplates", Sensors, 8(2), pp. 1048-1069 (2008).

15. Moghimi Zand, M. and Ahmadian, M.T. "Application of homotopy analysis method in studying dynamic pull-in instability of microsystems", Mech. Res. Commun., 36(7), pp. 851-858 (2009).

16. Moghimi Zand, M., Ahmadian, M.T. and Rashidian, B. "Semi-analytic solutions to nonlinear vibrations of microbeams under suddenly applied voltages", $J$. Sound Vib., 325(1), pp. 382-396 (2009).

17. Maleki, M., Tonekaboni, S.A.M. and Abbasbandy, S. "A homotopy analysis solution to large deformation of beams under static arbitrary distributed load", Appl. Math. Model., 38(1), pp. 355-368 (2014).

18. Sedighi, H.M., Koochi, A., Daneshmand, F. and Abadyan, M. "Non-linear dynamic instability of a double-sided nano-bridge considering centrifugal force and rarefied gas flow", Int. J. Non. Linear. Mech., 77, pp. 96-106 (2015).

19. Farrokhabadi, A., Mokhtari, J., Rach, R. and Abadyan, M. "Modeling the influence of the Casimir force on the pull-in instability of nanowire-fabricated nanotweezers", Int. J. Mod. Phys. B, 29(2), p. 1450245 (2015).

20. Ramezani, A., Alasty, A. and Akbari, J. "Effects of rotary inertia and shear deformation on nonlinear free vibration of microbeams", J. Vib. Acoust., 128(5), pp. 611-615 (2006).

21. Asghari, M., Kahrobaiyan, M.H. and Ahmadian, M.T. "A nonlinear Timoshenko beam formulation based on the modified couple stress theory", Int. J. Eng. Sci., 48(12), pp. 1749-1761 (2010).

22. Das, K. and Batra, R.C. "Pull-in and snap-through instabilities in transient deformations of microelectromechanical systems", J. Micromechanics Microengineering, 19(3), p. 35008 (2009).
23. Zand, M.M. and Ahmadian, M.T. "Application of homotopy analysis method in studying dynamic pullin instability of microsystems", Mech. Res. Commun., 36(7), pp. 851-858 (2009).

24. Ouakad, H.M. and Younis, M.I. "The dynamic behavior of MEMS arch resonators actuated electrically", Int. J. Non. Linear. Mech., 45(7), pp. 704-713 (2010).

25. Alipour, A., Zand, M.M. and Daneshpajooh, H. "Analytical solution to nonlinear behavior of electrostatically actuated nanobeams incorporating van der Waals and Casimir forces", Sci. Iran., 22(3), pp. 1322-1329 (2015).

26. Moghimi Zand, M. and Ostadi Moghaddam, A. "Pull-in instability and vibrations of a beam microgyroscope", J. Appl. Mech., 45(1), pp. 29-34 (2014).

27. Krylov, S., Ilic, B.R. and Lulinsky, S. "Bistability of curved microbeams actuated by fringing electrostatic fields", Nonlinear Dyn., 66(3), pp. 403-426 (2011).

28. Zand, M.M. "The dynamic pull-in instability and snapthrough behavior of initially curved microbeams", Mech. Adv. Mater. Struct., 19(6), pp. 485-491 (2012).

29. Stanciulescu, I., Mitchell, T., Chandra, Y., Eason, T. and Spottswood, M. "A lower bound on snap-through instability of curved beams under thermomechanical loads", Int. J. Non. Linear. Mech., 47(5), pp. 561-575 (2012).

30. Albers, B., Continuous Media with Microstructure, Springer (2010).

31. Zand, M.M. and Ahmadian, M.T. "Vibrational analysis of electrostatically actuated microstructures considering nonlinear effects", Commun. Nonlinear Sci. Numer. Simul., 14(4), pp. 1664-1678 (2009).

32. Zand, M.M. and Ahmadian, M.T. "Characterization of coupled-domain multi-layer microplates in pull-in phenomenon, vibrations and dynamics", Int. J. Mech. Sci., 49(11), pp. 1226-1237 (2007).

33. Mojahedi, M., Zand, M.M. and Ahmadian, M.T. "Investigation of the oscillatory behavior of elctrostatically-actuated microbeams", in ASME 2010 International Mechanical Engineering Congress and Exposition, pp. 619-626 (2010).

34. Zand, M.M., Ahmadian, M.T. and Rashidian, B. "Semi-analytic solutions to nonlinear vibrations of microbeams under suddenly applied voltages", J. Sound Vib., 325(1), pp. 382-396 (2009).

35. Chang, C.-S. and Hodges, D. "Vibration characteristics of curved beams", J. Mech. Mater. Struct., 4(4), pp. 675-692 (2009).

36. Abdulla, S.M.C., Yagubizade, H. and Krijnen, G.J.M. "Analysis of resonance frequency and pull-in voltages of curled micro-bimorph cantilevers", J. Micromechanics Microengineering, 22(3), p. 35014 (2012).

37. Ouakad, H.M., Younis, M.I., Alsaleem, F.M., Miles, 
R. and Cui, W. "The static and dynamic behavior of MEMS arches under electrostatic actuation", in ASME 2009 International Design Engineering Technical Conferences and Computers and Information in Engineering Conference, pp. 607-616 (2009).

38. Kuang, J.-H. and Chen, C.-J. "Dynamic characteristics of shaped micro-actuators solved using the differential quadrature method", J. Micromechanics Microengineering, 14(4), p. 647 (2004).

39. Abdel-Rahman, E.M., Younis, M.I. and Nayfeh, A.H. "Characterization of the mechanical behavior of an electrically actuated microbeam", J. Micromechanics Microengineering, 12(6), p. 759 (2002).

40. Younis, M.I., Modeling and Simulation of Microelectromechanical Systems in Multi-Physics Fields, Citeseer (2004).

41. Nayfeh, A.H. and Younis, M.I. "A new approach to the modeling and simulation of flexible microstructures under the effect of squeeze-film damping", J. Micromechanics Microengineering, 14(2), p. 170 (2004).

42. Nayfeh, A.H., Problems in Perturbation, John Wiley, New York (1985).

43. Liao, S.J., The Proposed Homotopy Analysis Technique for the Solution of Nonlinear Problems, Shanghai Jiao Tong University, China (1992).

44. Liao, S., Beyond Perturbation: Introduction to the Homotopy Analysis Method, CRC press (2003).

45. Daneshpajooh, H. and Zand, M.M. "Semi-analytic solutions to oscillatory behavior of initially curved micro/nano systems", J. Mech. Sci. Technol., 29(9), pp. 3855-3863 (2015).

46. Rosinger, H.E. and Ritchie, I.G. "On Timoshenko's correction for shear in vibrating isotropic beams", $J$. Phys. D. Appl. Phys., 10(11), p. 1461 (1977).

47. Komzsik, L., Applied Calculus of Variations for Engineers, CRC Press (2014).

48. Reddy, J.N., Energy Principles and Variational Methods in Applied Mechanics, John Wiley \& Sons (2002).

49. Ramezani, A., Alasty, A. and Akbari, J. "Effects of rotary inertia and shear deformation on nonlinear free vibration of microbeams", J. Vib. Acoust., 128(5), p. 611 (2006).

50. Tilmans, H.A.C. and Legtenberg, R. "Electrostatically driven vacuum-encapsulated polysilicon resonators: Part II. Theory and performance", Sensors Actuators A Phys., 45(1), pp. 67-84 (1994).

51. Tajalli, S.A., Moghimi Zand, M. and Ahmadian, M.T. "Effect of geometric nonlinearity on dynamic pull-in behavior of coupled-domain microstructures based on classical and shear deformation plate theories", Eur. J. Mech. A/Solids, 28(5), pp. 916-925 (2009).

\section{Appendix A}

Coefficient $a_{20}-a_{41}$ are presented as follows:

$$
\begin{aligned}
& a_{20}=I_{33}+N \frac{I_{33}}{F_{11}}, \\
& a_{21}=-\left(I_{33} \frac{m}{F_{11}}+m_{2}+m_{2} \frac{N}{F_{11}}\right), \\
& a_{22}=m-\frac{2 a m_{2}}{F_{11} d^{3}}-\frac{b m_{2}}{F_{11} d^{2}}, \\
& a_{23}=\frac{2 I_{33} a}{F_{11} d^{3}}+\frac{b I_{33}}{F_{11} d^{2}}-N \\
& a_{24}=\frac{6 I_{33} a}{F_{11} d^{4}}+\frac{2 b I_{33}}{F_{11} b^{3}}, \quad a_{25}=\frac{2 I_{33}}{F_{11} d^{3}}\left(\frac{3 a}{b}+b\right), \\
& a_{26}=\frac{6 I_{33}}{F_{11} d^{4}}\left(\frac{4 a}{d}+b\right), \quad a_{27}=\frac{3 I_{33}}{F_{11} d^{4}}\left(\frac{4 a}{d}+b\right), \\
& a_{28}=-\frac{2 m_{2}}{F_{11} d^{3}}\left(\frac{3 a}{d}+b\right) \\
& a_{29}=-\frac{2 m_{2}}{F_{11} d^{3}}\left(\frac{3 a}{d}+b\right), \\
& a_{30}=-\frac{6 m_{2}}{F_{11} d^{4}}\left(\frac{4 a}{d}+b\right), \\
& a_{31}=\frac{3 m_{2}}{F_{11} d^{4}}\left(\frac{4 a}{d}+b\right) \\
& a_{32}=\frac{m_{0} m_{2}}{F_{11}}, \quad a_{33}=-\frac{2 a}{d^{3}}-\frac{b}{d^{2}}, \\
& a_{34}=-\frac{3 a}{d^{4}}-\frac{b}{d^{3}}, \quad a_{35}=-\frac{4 a}{d^{5}}-\frac{b}{d^{4}}, \\
& a_{36}=-\frac{a}{d^{2}}-\frac{b}{d}, \quad a_{37}=\frac{12 I_{33}}{F_{11} d^{5}}\left(\frac{5 a}{d}+b\right), \\
& a_{38}=\frac{4 I_{33}}{F_{11} d^{5}}\left(\frac{5 a}{d}+b\right), \\
& a_{39}=-\frac{12 m_{2}}{F_{11} d^{5}}\left(\frac{5 a}{d}+b\right), \\
& a_{40}=-\frac{4 m_{2}}{F_{11} d^{5}}\left(\frac{5 a}{d}+b\right), \quad a_{41}=-\frac{5 a}{d^{6}}-\frac{b}{d^{5}},
\end{aligned}
$$

where $a, b, I_{33}$, and $F_{11}$ are defined as below:

$$
a=\frac{1}{2} b \varepsilon, \quad b=\frac{1}{2} \beta \varepsilon, \quad I_{33}=E I, \quad F_{11}=K A G .
$$




\section{Appendix B}

Coefficient $b_{1}-b_{24}$ are presented as follows:

$$
\begin{aligned}
& b_{1}=\left(-I_{33} \frac{m_{0}}{F_{11}}-m_{2}\right) \times \frac{1}{m_{0} L^{2}}, \\
& b_{2}=-\frac{m_{2}}{F_{11} d^{2}}\left(\frac{2}{d}+b\right) \times \frac{1}{m_{0}}, \\
& b_{3}=\frac{I_{33}}{F_{11} d^{2}}\left(\frac{2}{d}+b\right) \times \frac{L^{2}}{E I} \\
& b_{4}=\frac{N_{s}}{F_{11}}+\frac{E A d^{2}}{2 L^{2} F_{11}} \int_{0}^{1}\left(\frac{\partial \hat{w}}{\partial \hat{x}}\right)^{2} d \hat{x} \\
& b_{5}=-\frac{N_{s} m_{2}}{m L^{2} F_{11}}-\frac{E A d^{2} m_{2}}{2 L^{4} F_{11}} \int_{0}^{1}\left(\frac{\partial \hat{w}}{\partial \hat{x}}\right)^{2} d \hat{x}, \\
& b_{6}=-\frac{N_{s} L^{2}}{E I}-\frac{A d^{2}}{2 I} \int_{0}^{1}\left(\frac{\partial \hat{w}}{\partial \hat{x}}\right)^{2} d \hat{x}, \\
& b_{7}=a_{24} \times \frac{d L^{2}}{I_{33}}, \\
& b_{8}=a_{25} \times \frac{d L^{2}}{I_{33}}, \\
& b_{9}=a_{26} \times \frac{d^{2} L^{2}}{I_{33}} \\
& b_{10}=a_{27} \times \frac{d^{2} L^{2}}{I_{33}}, \\
& b_{11}=a_{37} \times \frac{d^{3} L^{2}}{I_{33}}, \\
& b_{12}=a_{38} \times \frac{d^{3} L^{2}}{I_{33}}, \\
& b_{13}=a_{33} \times \frac{L^{4}}{I_{33}}, \quad b_{14}=a_{34} \times \frac{d L^{4}}{I_{33}}, \\
& b_{15}=a_{35} \times \frac{d^{2} L^{4}}{I_{33}}, \quad b_{16}=a_{41} \times \frac{d^{3} L^{4}}{I_{33}}, \\
& b_{17}=a_{36} \times \frac{L^{4}}{I_{33} d}, \quad b_{18}=a_{32} \times \frac{I_{33}}{m^{2} L^{4}}, \\
& b_{19}=a_{28} \times \frac{d}{m}, \quad b_{20}=a_{29} \times \frac{d}{m}, \\
& b_{21}=a_{30} \times \frac{d^{2}}{m}, \quad b_{22}=a_{31} \times \frac{d^{2}}{m}, \\
& b_{23}=a_{39} \times \frac{d^{3}}{m}, \quad b_{24}=a_{40} \times \frac{d^{3}}{m} .
\end{aligned}
$$

\section{Appendix $\mathrm{C}$}

Coefficient $a_{1}-a_{16}$ are presented as follows:

$$
\begin{aligned}
& a_{1}=\frac{a_{1}^{\prime}+b_{2}^{\prime}}{a_{18}^{\prime}}, \quad a_{2}=\frac{b_{1}^{\prime}+a_{25}{ }^{\prime}}{a_{18}{ }^{\prime}}, \\
& a_{3}=\frac{a_{7}^{\prime}+a_{8}^{\prime}+a_{14}^{\prime}}{a_{18}{ }^{\prime}}, \quad a_{4}=\frac{a_{4}^{\prime}+a_{6}^{\prime}}{a_{18}{ }^{\prime}}, \\
& a_{5}=\frac{a_{11}{ }^{\prime}+a_{12}{ }^{\prime}+a_{16}{ }^{\prime}}{a_{18}{ }^{\prime}}, \quad a_{6}=\frac{a_{19}{ }^{\prime}}{a_{18}{ }^{\prime}}, \\
& a_{7}=\frac{a_{21}{ }^{\prime}}{a_{18}{ }^{\prime}}, \quad a_{8}=\frac{a_{23}{ }^{\prime}}{a_{18}{ }^{\prime}}, \\
& a_{9}=\frac{a_{20}{ }^{\prime}}{a_{18}{ }^{\prime}}, \quad a_{10}=\frac{a_{5}^{\prime}}{a_{18}{ }^{\prime}}, \\
& a_{11}=\frac{a_{24}{ }^{\prime}}{a_{18}{ }^{\prime}}, \quad a_{12}=\frac{a_{17^{\prime}}{ }^{\prime}}{a_{18^{\prime}}}, \\
& a_{13}=\frac{a_{2}{ }^{\prime}}{a_{18}{ }^{\prime}}, \quad a_{14}=\frac{a_{3}{ }^{\prime}+a_{13}{ }^{\prime}}{a_{18}{ }^{\prime}}, \\
& a_{15}=\frac{a_{9}{ }^{\prime}+a_{10^{\prime}}+a_{15}{ }^{\prime}}{a_{18}{ }^{\prime}}, \quad a_{16}=\frac{a_{22}{ }^{\prime}}{a_{18}{ }^{\prime}}, \\
& b_{1}^{\prime}=\left(\frac{N_{s}}{F_{11}}+1\right) \int_{0}^{1} \varphi \varphi^{(4)} d \hat{x} \\
& b_{2}^{\prime}=\int_{0}^{1} \varphi^{2} d \hat{x} \\
& a_{1}^{\prime}=\left(b_{1}-\frac{N_{s} m_{2}}{m L^{2} F_{11}}\right) \int_{0}^{1} \varphi \varphi^{\prime \prime} d \hat{x} \\
& a_{2}^{\prime}=b_{2} \int_{0}^{1} \varphi^{2} d \hat{x}, \quad a_{3}^{\prime}=b_{3} \int_{0}^{1} \varphi \varphi^{\prime \prime} d \hat{x} \\
& a_{4}^{\prime}=\frac{E A d^{2}}{2 L^{2} F_{11}} \int_{0}^{1} \varphi \varphi^{(4)}\left(\int_{0}^{1}\left(\varphi^{\prime}\right)^{2} d \hat{x}\right) d \hat{x}, \\
& a_{5}^{\prime}=-\frac{E A d^{2} m_{2}}{2 L^{4} F_{11}} \int_{0}^{1} \varphi \varphi^{\prime \prime}\left(\int_{0}^{1}\left(\varphi^{\prime}\right)^{2} d \hat{x}\right) d \hat{x} \\
& a_{6}^{\prime}=-\frac{A d^{2}}{2 I} \int_{0}^{1} \varphi \varphi^{\prime \prime}\left(\int_{0}^{1}\left(\varphi^{\prime}\right)^{2} d \hat{x}\right) d \hat{x} \\
& a_{7}^{\prime}=b_{7} \int_{0}^{1} \varphi\left(\varphi^{\prime}\right)^{2} d \hat{x}, \quad a_{8}^{\prime}=b_{8} \int_{0}^{1} \varphi^{2} \varphi^{\prime \prime} d \hat{x} \\
& a_{9}^{\prime}=b_{9} \int_{0}^{1} \varphi^{2}\left(\varphi^{\prime}\right)^{2} d \hat{x}, \quad a_{10}^{\prime}=b_{10} \int_{0}^{1} \varphi^{3} \varphi^{\prime \prime} d \hat{x} \\
& a_{11}^{\prime}=b_{11} \int_{0}^{1} \varphi^{3}\left(\varphi^{\prime}\right)^{2} d \hat{x}, \quad a_{12}{ }^{\prime}=b_{12} \int_{0}^{1} \varphi^{4} \varphi^{\prime \prime} d \hat{x}
\end{aligned}
$$




$$
\begin{aligned}
& a_{13}{ }^{\prime}=b_{13} \int_{0}^{1} \varphi^{2} d \hat{x}, \quad a_{14}{ }^{\prime}=b_{14} \int_{0}^{1} \varphi^{3} d \hat{x}, \\
& a_{15}{ }^{\prime}=b_{15} \int_{0}^{1} \varphi^{4} d \hat{x}, \quad a_{16}{ }^{\prime}=b_{16} \int_{0}^{1} \varphi^{5} d \hat{x}, \\
& a_{17}{ }^{\prime}=b_{17} \int_{0}^{1} \varphi d \hat{x}, \quad a_{18}{ }^{\prime}=b_{18} \int_{0}^{1} \varphi^{2} d \hat{x}, \\
& a_{19^{\prime}}=b_{19} \int_{0}^{1} \varphi^{3} d \hat{x}, \quad a_{20}{ }^{\prime}=b_{20} \int_{0}^{1} \varphi^{3} d \hat{x}, \\
& a_{21}{ }^{\prime}=b_{21} \int_{0}^{1} \varphi^{4} d \hat{x}, \quad a_{22}{ }^{\prime}=b_{22} \int_{0}^{1} \varphi^{4} d \hat{x}, \\
& a_{23}{ }^{\prime}=b_{23} \int_{0}^{1} \varphi^{5} d \hat{x}, \quad a_{24}{ }^{\prime}=b_{24} \int_{0}^{1} \varphi^{5} d \hat{x}, \\
& a_{25}{ }^{\prime}=-\frac{N_{s} L^{2}}{E I} \int_{0}^{1} \varphi \varphi^{\prime \prime} d \hat{x} .
\end{aligned}
$$

\section{Appendix D}

The terms $u_{1}(t)$, and $u_{2}(t)$ are presented in Box I and $\omega_{1}, \omega_{2}, v_{1}$ and $v_{2}$ are presented as follows:

$$
\begin{aligned}
\omega_{1}= & \frac{\left(-a_{9} \omega^{2}+2 a_{3}\right) V^{4} a_{12} h}{v^{2}\left(v^{2}-\omega^{2}\right) \omega^{2}}, \\
v_{1}= & \frac{\left(-a_{9} v^{2}+2 a_{3}\right) V^{4} a_{12} h}{\omega^{2} v^{2}\left(v^{2}-\omega^{2}\right)} . \\
\omega_{2}= & \frac{1}{4} \frac{1}{v^{4} \omega^{4}\left(v^{6}-3 v^{4} \omega^{2}+3 v^{2} \omega^{4}-\omega^{6}\right)} \\
& \left(V ^ { 4 } a _ { 1 2 } h \left(4 V^{4} a_{12} a_{9}^{2} h v^{2} \omega^{2}+4 a_{9} h v^{6} \text { omega }{ }^{4}\right.\right. \\
& -8 a_{9} h v^{4} \omega^{6}+4 a_{9} h v^{2} \omega^{8}+7 V^{2} a_{12} a_{16} h v^{4} \omega^{2} \\
& -4 V^{2} a_{12} a_{16} h v^{2} \omega^{4}+6 V^{2} a_{12} a_{16} h \omega^{6} \\
& -V^{2} a_{12} a_{7} h v^{4} \omega^{2}-2 V^{2} a_{12} a_{7} h v^{2} \omega^{4} \\
& +4 a_{9} v^{6} \omega^{4}-8 a_{9} v^{4} \omega^{6}+4 a_{9} v^{2} \omega^{8}
\end{aligned}
$$

$$
\begin{aligned}
& -8 V^{4} a_{12} a_{3} a_{9} h v^{2}-8 V^{4} a_{12} a_{3} a_{9} h \omega^{2} \\
& -8 a_{3} h v^{6} \omega^{2}+16 a_{3} h v^{4} \omega^{4}-8 a_{3} h v^{2} \omega^{6} \\
& -15 V^{2} a_{12} a_{15} h v^{4}+24 V^{2} a_{12} a_{15} h v^{2} \omega^{2} \\
& -18 V^{2} a_{12} a_{15} h \omega^{4}+7 a_{10} a_{12} h v^{4} \omega^{2} \\
& -4 a_{10} a_{12} h v^{2} \omega^{4}+6 a_{10} a_{12} h \omega^{6}-8 a_{3} v^{6} \omega^{2} \\
& +16 a_{3} v^{4} \omega^{4}-a_{3} v^{2} \omega^{6}+16 V^{4} a_{12} a_{3}^{2} h \\
& -15 a_{12} a_{4} h v^{4}+24 a_{12} a_{4} h v^{2} \omega^{2} \\
& \left.\left.-18 a_{12} a_{4} h \omega^{4}\right)\right),
\end{aligned}
$$$$
v_{2}=\frac{1}{4} \frac{1}{v^{4} \omega^{4}\left(v^{6}-3 v^{4} \omega^{2}+3 v^{2} \omega^{4}-\omega^{6}\right)}
$$$$
\left(V ^ { 4 } a _ { 1 2 } h \left(4 V^{4} a_{12} a_{9}^{2} h v^{2} \omega^{2}+4 a_{9} h v^{8} \text { omega }^{2}\right.\right.
$$$$
-8 a_{9} h v^{6} \omega^{4}+4 a_{9} h v^{4} \omega^{6}+6 V^{2} a_{12} a_{16} h v^{6}
$$$$
-4 V^{2} a_{12} a_{16} h v^{4} \omega^{2}+7 V^{2} a_{12} a_{16} h v^{2} \omega^{4}
$$$$
-2 V^{2} a_{12} a_{7} h v^{4} \omega^{2}-V^{2} a_{12} a_{7} h v^{2} \omega^{4}
$$$$
+4 a_{9} v^{8} \omega^{2}-8 a_{9} v^{6} \omega^{4}+4 a_{9} v^{4} \omega^{6}
$$$$
-8 V^{4} a_{12} a_{3} a_{9} h v^{2}-8 V^{4} a_{12} a_{3} a_{9} h \omega^{2}
$$$$
-8 a_{3} h v^{6} \omega^{2}+16 a_{3} h v^{4} \omega^{4}-8 a_{3} h v^{2} \omega^{6}
$$$$
-18 V^{2} a_{12} a_{15} h v^{4}+24 V^{2} a_{12} a_{15} h v^{2} \omega^{2}
$$$$
-15 V^{2} a_{12} a_{15} h \omega^{4}+6 a_{10} a_{12} h v^{6}
$$$$
-4 a_{10} a_{12} h v^{4} \omega^{2}+7 a_{10} a_{12} h n^{2} \omega^{4}
$$$$
-8 a_{3} v^{6} \omega^{2}+16 a_{3} v^{4} \omega^{4}-8 a_{3} v^{2} \omega^{6}
$$$$
+16 V^{4} a_{12} a_{3}^{2} h-18 a_{12} a_{4} h v^{4}+24 a_{12} a_{4} h v^{2} \omega^{2}
$$$$
\left.\left.-15 a_{12} a_{4} h \omega^{4}\right)\right) \text {. }
$$

$$
\begin{aligned}
& u_{1}(t)=\frac{\frac{h a_{12} V^{2} \cos (v t) \omega^{2}}{v^{2}-\omega^{2}}-\frac{h a_{12} V^{2} \cos (\omega t) v^{2}}{v^{2}-\omega^{2}}+h_{12} V^{2}}{\omega^{2} v^{2}}, \\
& u_{2}(t)=\frac{\frac{h a_{12} V^{2}(h+1) \cos (v t) \omega^{2}}{v^{2}-\omega^{2}}-\frac{h a_{12} V^{2}(h+1) \cos (\omega t) v^{2}}{v^{2}-\omega^{2}}+h^{2} a_{12} V^{2}+h a_{12} V^{2}}{\omega^{2} v^{2}} .
\end{aligned}
$$




\section{Biographies}

Masoud Molaaghaie Roozbahani received a BS degree in Mechanical Engineering from Semnan University, Semnan, Iran, in 2012, and is currently an MS degree student in the Department of Mechanical Engineering at University of Tehran, Iran. His research interests include nonlinear vibration of micro/nano systems, MEMS, bio-medical engineering, and smart materials.

Navid Heydarzadeh Arani received a BS degree in Mechanical Engineering from University of Kashan, Kashan, Iran, in 2012, and is currently an MS degree student in the Department of Mechanical Engineering at University of Tehran, Iran. His research interests include solid mechanics, micro structure, and smart materials.
Mahdi Moghimi Zand is a Faculty Member in the Department of Mechanical Engineering at University of Tehran, Iran. He has received numerous academic awards, including the Young Professor Grant and the Fellowship of the National Elite Foundation. Dr. Moghimi Zand's research is multi-disciplinary and includes vibrations, MEMS, bio-medical engineering, and cancer engineering.

Mahmood Mousavi Mashhadi is a Faculty Member in the Department of Mechanical Engineering at University of Tehran, Iran. He received the BS degree in Physics and Mathmatics from Union College, USA, in 1977, and the MS and PhD degrees in Mechanical Engineering from University of Arkansas, USA, in 1980 and 1984, respectively. Professor Mousavi Mashhadi's research interests are composite structures, micro/nano structures, and computational mechanics. 